\title{
Research on Effective Evaluation of POL Support System Based on the Internet of Things and Cloud Theory
}

\author{
Chun Yuan ${ }^{1,2, *}$, Qidong Yong ${ }^{1}$, Yang Chen ${ }^{1}$, Ming Long ${ }^{1}$, Heng Li $^{1}$, Ke Zhu ${ }^{1}$, Qi Zhang ${ }^{1}$, Hongchun \\ $\mathrm{Su}^{2}$ and Yun $\mathrm{Chen}^{1}$ \\ ${ }^{I}$ Department of POL Application and Management, Logistic Engineering University, Chongqing, 400035, China \\ ${ }^{2}$ Deparment of Electric Power, Chongqing Communication Institute, Chongqing, 400035, China
}

\begin{abstract}
Many factors should be studied during the course of the evaluation of POL support efficiency, especially the evaluation of POL support efficiency based on the internet of the things. To avoid uncertainty and randomness of correlative assessment works, simplifying the complexity of effectiveness evaluation of POL support system based on the internet of things, the principles of cloud theory is adopted. The effectiveness evaluation model of POL support system is established based on cloud theory in this paper. After the analysis of the influence factors of the evaluation of POL support based on the internet of the things, a three-level index system is established, and the course of the cloud evaluation is described step by step. After the precise calculation, the total result of the evaluation can be obtained. From the final result, it can be learned that the POL support efficiency based on the internet of things is excellent. The conclusion can be used in the evaluation of the POL support efficiency based on the internet of things and the cloud theory.
\end{abstract}

Keywords: Cloud theory, efficiency, evaluation, POL support, the internet of things.

\section{INTRODUCTION}

The new POL support way is based on the internet of things. Whether the new POL support way is fit for the requirement or not, the best way is to evaluate the POL support efficiency based on the internet of the things. The evaluation of the POL support efficiency has many methods, such as fuzzy comprehensive evaluation, grey evaluation, Delphi evaluation, FHW evaluation, Analytical Hierarchy Process, etc.

Fuzzy comprehensive evaluation method is a comprehensive evaluation method based on fuzzy mathematics. The comprehensive evaluation method based on the membership degree theory in fuzzy mathematics by qualitative and quantitative evaluation, namely by the things or objects, many factors make an overall evaluation by fuzzy mathematics. It has a clear result, the characteristics of a strong system, it can solve the fuzzy problem solving, which are difficult to quantify and suitable for all kinds of nondeterministic problem. In the method of the fuzzy assessment, the related factors of the POL support efficiency are almost uncertain, just like "black box".

The grey evaluation theory and method based on the grey system theory, the evaluation object is located at the first stage of evaluation. The main characteristic of the grey evaluation is that it can perform multiple processes. In the gray evaluation, the evaluation process can be recycled; the evaluation result of process can be used as the input data of evaluation process. Therefore, the grey multi-level evaluation can meet the requirements of complex system evaluation. Grey evaluation and AHP evaluation are always used in combination.

Delphi method is also called the expert investigation method, and that is a kind of means of communication to solve the problem individually sent to each expert. Then advice of the experts will be recovered, and the comprehensive views will be sorted out. The general opinion and prediction problems then will be used as a feedback to the expert as an advice of the experts, once again, on the basis of comprehensive revisions to their original opinion, then the result will be summarized. The process will be performed repeatedly and the more consistent decision methods and prediction results will be gradually obtained. The main drawback of Delphi evaluation is that it has a very complex process and takes a long time.

FHW (fuzzy, grey, element) evaluation is a new assessment method of decision-making by Chinese professor He. The method is the improvement and development of Delphi method, it combines the Delphi method, BS method (brainstorming) and the advantages of the KT method. The emerging discipline of the way of thinking, such as fuzzy mathematics, the grey system theory and the matter element analysis, which can be used with associative thinking, while the Delphi method consulting table to FHW consulting table, 
by a number of expert consultation (order, judgment, scoring) into a fuzzy, grey matter element. The method makes the combination of fuzzy evaluation and grey analysis, a provenance analysis.

AHP is the method that makes the element related of decision-making broken down into objectives, standards, programs and other levels, decision-making method then will be based on qualitative and quantitative analysis of the basis. The characteristics of the analytic hierarchy process are the basis for the analysis of complex decision problems in nature and its inherent relationship between factors and so on. Hierarchical analysis method is used to simulate the human decision-making method with qualitative color. Quantitative data are few, qualitative components are multiple, and then the results are largely accepted but there remains a little criticism.

These methods can be used to solve most problems of the evaluation when the influence factors are uncertain. With the application of the internet of the things in the POL support, the object of the net can be orientated in the obvious time, and then the POL support can be realized in the real time, the real amount and the real location. While on the basis of the internet of the things, the certain factors of the POL support are increased, and uncertain factors are decreased, to deal with the change of the POL support efficiency evaluation, the methods mentioned above could not solve both the uncertainty and certainty, the new method should be studied.

Cloud theory is a qualitative concept expressed by language and its quantitative expression, its an uncertain conversion model between two kinds of uncertainty, it mainly reflects the universe and human knowledge in the concept of fuzzy (boundary: the ratio of) and random (probability), cloud model of the fuzzy and the random are fully integrated together. The research on natural language in the basic language value contains the universal law of uncertainty, the range of quantitative data and distribution may be expressed qualitatively from linguistic information, then the appropriate value may be also converted into qualitative language from the exact numerical effectively.

That is to say, cloud theory can be used to reflect a concept of fuzziness and randomness, and fuzziness and randomness by fusion can be formed between quantitative and qualitative mapping, then the cloud theory is described as an edge theory which is based on the traditional fuzzy set theory and probability and statistics

The cloud theory is chosen.

\section{MATERIALS AND METHODOLOGY}

\section{A. Theoretical Model}

Assume that $\square$ is an exact numeric representation with the domain, and the corresponding qualitative concept is $\square$, then for any element of the domain, there is a stable tendency random number $y=\mu_{A}(v)$, which is called the membership of $\mu$ for $A$. The distribution of $\nu$ in the domain of $V$ is called membership cloud. Cloud model is the mapping from $V$ to the interval $[0,1]$ :

$$
\text { (. ) : } \rightarrow[0,1] \forall . \in,, \rightarrow \text {. (. ) }
$$

Numerical characteristics of cloud are usually represented by. ., . and ., which reflect the qualitative concept of quantitative characteristics. Among them, the

is the center of gravity of the cloud, which means that the corresponding fuzzy concept expectations; the . is used to express the concept of blurring the range of values and the vague concept of domains that can be accepted by the number of elements, the greater the .., the stronger the ambiguity of the concept is; the . is a measure of the thickness of the cloud, the maximum thickness of the entire cloud, which means that the degree of dispersion of the cloud droplets. Three values of cloud features can be graphed in Fig. (1).

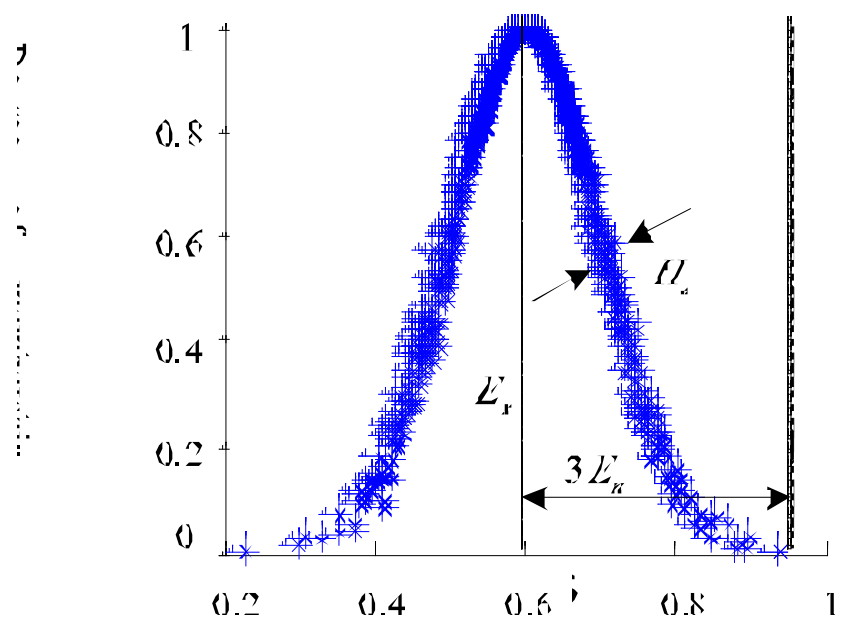

Fig. (1). The numerical value characteristic of the cloud.

Cloud center-of-gravity can be described as $\square$, in the formula, "a" is used to indicate the position of the cloud center-of-gravity, "b" is used to represent the height of the cloud center-of-gravity. $\square$ reflects the corresponding fuzzy concept information center value, which is the cloud center of gravity position [1].

\section{B. Steps of Model Calculation}

\section{(1) Cloud Eigen Values of the Third-level Indexes}

Assume that $\square$ sets of relevant data are composed of decision matrix and the nonnumeric representation of an indicator can be used to represent one cloud model. In the model, $\square$ and $\square$ can be described as follows:

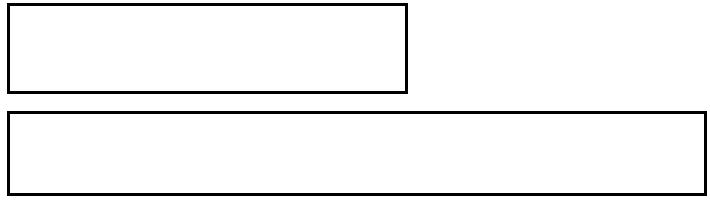

Each language value based indicators can also be used to represent a cloud model, $E_{x}$ and $E_{n}$ can be described as follows: 


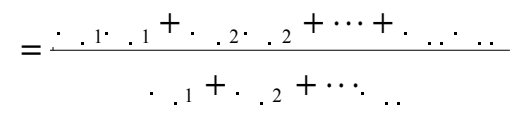

$$
\begin{aligned}
& ={ }_{.1}+{ }_{.2}+\cdots . .
\end{aligned}
$$

In the above formulas, when the index has the value of number, . is the value of the index, when the index has the value of the language, $\square$ are the expectations of the index, is entropy of each indicator of the cloud model [2].

\section{(2) The Index Weight of Each Level}

There are several methods for determining the index weights are more, such as expert ratings, information entropy method. The paper used expert ratings to determine the levels of index weight values.

\section{(3) Changes in Cloud Center-of-Gravity}

Assume that ideally $\square$ dimensional of the integrated cloud center-of-gravity position vector is \begin{tabular}{|l|l}
\hline & , cloud center-of-gravity height vector is \\
\hline & $\square$ is the corresponding weight of the
\end{tabular} index, the cloud center-of-gravity vector in the ideal state can be expressed as . From above, $\square$ dimensional of the integrated cloud center-of-gravity can be obtained, after being normalized, a new vector will be obtained as vector $\square$ is determined by

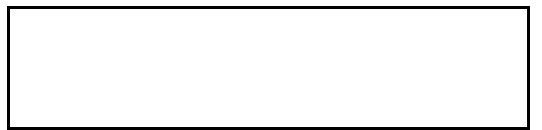

After being normalized, the value of each index vector is multiplied by its weight, and then the results are summed, the weighted deviation $\square$ can be obtained as follows:

Thus, the degree of deviation $\square$ can be used to measure the differences between the integrated cloud center-ofgravity of the weighted state and the cloud center-of-gravity of the ideal state in $\square$ dimensional, the smaller the value of $\square$ is, the closer it is to the ideal state.

\section{(4) Determined Comment Set}

In this paper, reviews set $U=\left(u_{1}, u_{2}, \cdots, u_{11}\right)=$ (none, much poorer, poorer, very poor, poor, fair, good, very good, much better, and excellent) are used, and obtained the successive language value of rulers, then the value of each comment can be achieved by the cloud model, a cloud generator will be generated. For example, for comment as "much good" formed by the cloud is taken as $(0.8,0.15$,
$0.005)$, the cloud model can be expressed as shown in Fig. (2).

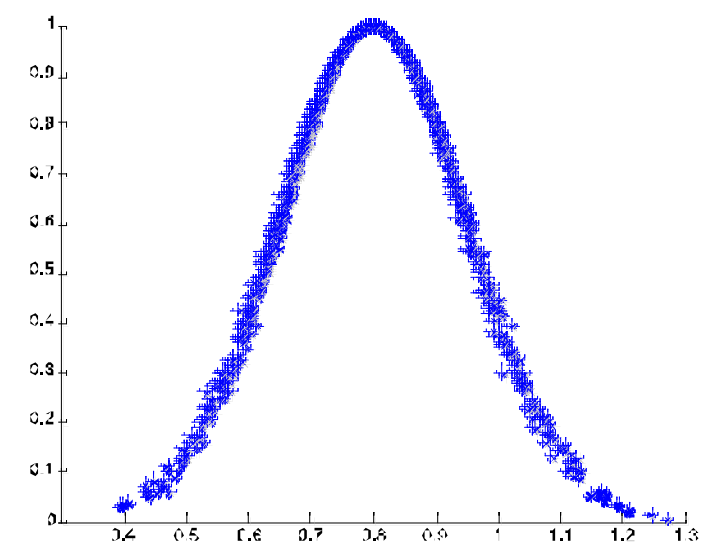

Fig. (2). Comment value of very good state implemented by cloud model.

Cloud generator composed of reviews set is shown as in Fig. (3).

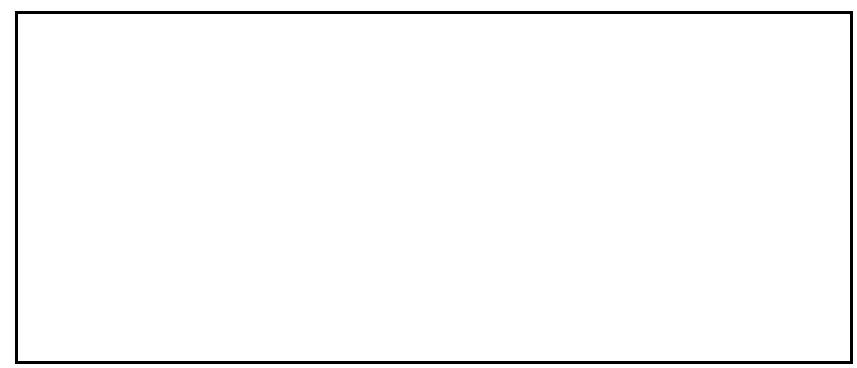

Fig. (3). Cloud generator of comment set.

The calculated value of $\square$ is an input in the cloud generator, if the corresponding value of a comment cloud object is much greater than the value of the other comment, the comment may be an evaluation value; if the value of $\square$ is corresponding to the two reviewed cloud objects, and the difference is less then the principles of integrated cloud should be applied to produce a new cloud object and the new value of the expectation will be taken as the evaluation result.

\section{(5) Attribution Degree Evaluation of the Second-level Indexes}

Based on the weighted deviation value of $\square$, attribution degree of the second-level indexes can be obtained from the formula (8).

$e=1-|\delta|$

\section{(6) Complex Evaluation of POL Support Efficiency}

According to second-level indexes attribution degree and the weight of second-level indexes by calculation, the value of first-level indexes can be obtained as follows:

$$
A_{i}=\sum_{j=1}^{k} w_{i j} e_{i j} \quad i=1,2
$$


In the formula (9), $i$ is response for the first-level indicators; $j$ is response for the second-level indicator under the first-level indicator $i ; k$ is the number of the second-level indexes. Finally, according to the formula (9), POL support efficiency integrated assessment value can be obtained.

\section{CALCULATION AND RESULTS}

According to the impact of the internet of things to the POL support, the weight of the third-level should be determined first. The standard of the scoring criteria is in the Table 1.

Table 1. Standard and range of evaluation score of the third level indexes.

\begin{tabular}{|c|c|}
\hline Status of the index & Range \\
\hline \hline excellent & $0.90 \sim 1.0$ \\
\hline better & $0.80 \sim 0.89$ \\
\hline good & $0.70 \sim 0.79$ \\
\hline ordinary & $0.60 \sim 0.69$ \\
\hline bad & $0 \sim 0.59$ \\
\hline
\end{tabular}

After the score according the Table 1, the weight of 26 third-level indexes can be got as Table 2 .

The cloud model features of the third-level indexes can be calculated by the formula (2) and (3), the results are shown in Table 3.

Application the method of DELPH, the weight of each level index can be got as the Table 4 .

Since the attribute values of the third-level indexes are in the range $[0,1]$, all the values of the third-level indexes are 1 in the ideal state. For the second-level, the weighted composite cloud could be used to represent according to the corresponding third-level index, according to the formula of $\square$, weighted composite cloud center-of-gravity vector in the ideal state and the actual integrated center-of-gravity vector cloud can be obtained [3].

Such as management effectiveness index, which can be represented by weighted composite cloud composed of four third-level indexes, then the value of weighted composite cloud gravity vector in the ideal state can be obtained, as below:

$$
\begin{aligned}
& G_{11}^{0}=\left[W_{111} \times 1, W_{112} \times 1, W_{113} \times 1, W_{114} \times 1\right] \\
& =\left[\begin{array}{llll}
0.035 & 0.078 & 0.042 & 0.033
\end{array}\right]
\end{aligned}
$$

Table 2. Attribute value of the third indexes.

\begin{tabular}{|c|c|c|c|c|c|c|c|c|c|c|c|c|c|}
\hline status & $\mathbf{A 1 1 1}$ & $\mathbf{A 1 1 2}$ & $\mathbf{A 1 1 3}$ & $\mathbf{A 1 1 4}$ & $\mathbf{A 1 2 1}$ & $\mathbf{A 1 2 2}$ & $\mathbf{A 1 3 1}$ & $\mathbf{A 1 3 2}$ & $\mathbf{A 2 1 1}$ & $\mathbf{A 2 1 2}$ & $\mathbf{A 2 1 3}$ & $\mathbf{A 2 2 1}$ & $\mathbf{A 2 2 2}$ \\
\hline \hline 1 & 0.89 & 0.83 & 0.86 & 0.80 & 0.84 & 0.82 & 0.85 & 0.80 & 0.90 & 0.80 & 0.85 & 0.90 & 0.86 \\
\hline 2 & 0.86 & 0.80 & 0.85 & 0.81 & 0.83 & 0.85 & 0.86 & 0.85 & 0.89 & 0.83 & 0.87 & 0.89 & 0.88 \\
\hline 3 & 0.85 & 0.79 & 0.82 & 0.89 & 0.80 & 0.80 & 0.80 & 0.82 & 0.86 & 0.80 & 0.85 & 0.88 & 0.85 \\
\hline 4 & 0.89 & 0.82 & 0.83 & 0.82 & 0.81 & 0.81 & 0.85 & 0.85 & 0.88 & 0.80 & 0.88 & 0.90 & 0.88 \\
\hline
\end{tabular}

\begin{tabular}{|c|c|c|c|c|c|c|c|c|c|c|c|c|c|}
\hline status & $\mathbf{A 2 3 1}$ & $\mathbf{A 2 3 2}$ & $\mathbf{A 3 1 1}$ & $\mathbf{A 3 1 2}$ & $\mathbf{A 3 2 1}$ & $\mathbf{A 3 2 2}$ & $\mathbf{A 3 2 3}$ & $\mathbf{A 4 1 1}$ & $\mathbf{A 4 1 2}$ & $\mathbf{A 4 1 3}$ & $\mathbf{A 4 2 1}$ & $\mathbf{A 4 2 2}$ & $\mathbf{A 4 2 3}$ \\
\hline \hline 1 & 0.80 & 0.85 & 0.86 & 0.92 & 0.89 & 0.81 & 0.81 & 0.88 & 0.82 & 0.88 & 0.82 & 0.80 & 0.80 \\
\hline 2 & 0.82 & 0.82 & 0.85 & 0.90 & 0.87 & 0.83 & 0.82 & 0.85 & 0.85 & 0.85 & 0.85 & 0.82 & 0.82 \\
\hline 3 & 0.79 & 0.80 & 0.81 & 0.88 & 0.83 & 0.80 & 0.80 & 0.81 & 0.80 & 0.85 & 0.82 & 0.80 & 0.80 \\
\hline 4 & 0.85 & 0.88 & 0.87 & 0.91 & 0.88 & 0.82 & 0.83 & 0.86 & 0.83 & 0.87 & 0.86 & 0.81 & 0.85 \\
\hline
\end{tabular}

\begin{tabular}{|c|c|c|c|c|c|c|c|c|c|c|c|c|c|}
\hline Index & A111 & A112 & A113 & A114 & A121 & A122 & A131 & A132 & A211 & A212 & A213 & A221 & A222 \\
\hline$E_{x}$ & 0.87 & 0.81 & 0.84 & 0.83 & 0.82 & 0.82 & 0.84 & 0.84 & 0.89 & 0.81 & 0.86 & 0.89 & 0.87 \\
\hline$E_{n}$ & 0.007 & 0.007 & 0.007 & 0.015 & 0.007 & 0.008 & 0.01 & 0.008 & 0.007 & 0.005 & 0.005 & 0.003 & 0.005 \\
\hline
\end{tabular}

Table 3. Expectation and entropy of the third indexes

\begin{tabular}{|c|c|c|c|c|c|c|c|c|c|c|c|c|c|}
\hline Index & A231 & A232 & A311 & A312 & A321 & A322 & A323 & A411 & A412 & A413 & A421 & A422 & A423 \\
\hline$E_{x}$ & 0.84 & 0.84 & 0.85 & 0.90 & 0.87 & 0.82 & 0.82 & 0.85 & 0.83 & 0.86 & 0.84 & 0.81 & 0.82 \\
\hline$E_{n}$ & 0.01 & 0.013 & 0.01 & 0.007 & 0.01 & 0.005 & 0.005 & 0.008 & 0.008 & 0.005 & 0.007 & 0.003 & 0.007 \\
\hline
\end{tabular}


Table 4. Weight of each level index.

\begin{tabular}{|c|c|c|c|c|c|}
\hline \multicolumn{2}{|c|}{ First-level } & \multicolumn{2}{|c|}{ Second-level } & \multicolumn{2}{|c|}{ Third-level } \\
\hline Index & Weight & Index & Weight & Index & Weight \\
\hline \multirow{8}{*}{$\begin{array}{c}\text { Support } \\
\text { efficiency } \\
\text { (A1) }\end{array}$} & \multirow{8}{*}{0.275} & \multirow{4}{*}{$\begin{array}{c}\text { Management } \\
\text { effectiveness (A11) }\end{array}$} & \multirow{4}{*}{0.178} & Allocate foresee (A111) & 0.035 \\
\hline & & & & Transfer visibility (A112) & 0.078 \\
\hline & & & & Store intelligent (A113) & 0.042 \\
\hline & & & & Fuel automation (A114) & 0.033 \\
\hline & & \multirow{2}{*}{$\begin{array}{c}\text { Economic } \\
\text { effectiveness (A12) }\end{array}$} & \multirow{2}{*}{0.025} & $\operatorname{cost}(\mathrm{A} 121)$ & 0.009 \\
\hline & & & & application (A122) & 0.016 \\
\hline & & \multirow{2}{*}{$\begin{array}{c}\text { Time effectiveness } \\
\text { (A13) }\end{array}$} & \multirow{2}{*}{0.072} & plan (A131) & 0.042 \\
\hline & & & & efficiency (A132) & 0.030 \\
\hline \multirow{7}{*}{$\begin{array}{c}\text { Network } \\
\text { Building (A2) }\end{array}$} & \multirow{7}{*}{0.275} & \multirow{3}{*}{$\begin{array}{l}\text { Network Function } \\
\text { (A21) }\end{array}$} & \multirow{3}{*}{0.125} & integrity (A211) & 0.045 \\
\hline & & & & reliability (A212) & 0.046 \\
\hline & & & & Network life (A213) & 0.034 \\
\hline & & \multirow{2}{*}{ Data Center (A22) } & \multirow{2}{*}{0.075} & criteria unify (A221) & 0.053 \\
\hline & & & & Self-keep (A222) & 0.022 \\
\hline & & \multirow{2}{*}{$\begin{array}{l}\text { Cloud platform } \\
\text { (A23) }\end{array}$} & \multirow{2}{*}{0.075} & The decision accuracy (A231) & 0.053 \\
\hline & & & & Self-keep (A232) & 0.022 \\
\hline \multirow{5}{*}{$\begin{array}{c}\text { Information } \\
\text { Security (A3) }\end{array}$} & \multirow{5}{*}{0.15} & \multirow{2}{*}{$\begin{array}{l}\text { Usable Function } \\
\text { (A31) }\end{array}$} & \multirow{2}{*}{0.075} & Node usable (A3111) & 0.039 \\
\hline & & & & Information usable (A312) & 0.036 \\
\hline & & \multirow{3}{*}{ integrality (A32) } & \multirow{6}{*}{0.175} & Data integrity (A321) & 0.035 \\
\hline & & & & Chain use ratio (A322) & 0.016 \\
\hline & & & & Network secret (A323) & 0.024 \\
\hline \multirow{6}{*}{$\begin{array}{l}\text { Information } \\
\text { value(A4) }\end{array}$} & \multirow{6}{*}{0.30} & \multirow{3}{*}{$\begin{array}{c}\text { Information } \\
\text { Relationship (A41) }\end{array}$} & & Node information sensitive (A411) & 0.055 \\
\hline & & & & Node information fusion (A412) & 0.055 \\
\hline & & & & Node information real time (A413) & 0.065 \\
\hline & & & & Information specialty (A421) & 0.034 \\
\hline & & $\begin{array}{l}\text { Information Usable } \\
\text { (A42) }\end{array}$ & 0.125 & information share (A422) & 0.046 \\
\hline & & & & information accuracy (A423) & 0.035 \\
\hline
\end{tabular}

The management effectiveness of the actual integrated cloud gravity vector is determined by the value of the expectation and the weight, as below:

$$
\begin{aligned}
& G_{11}=\left[W_{111} \times E_{x 1}, W_{112} \times E_{x 2}, W_{113} \times E_{x 3}, W_{114} \times E_{x 4}\right] \\
& =\left[\begin{array}{lll}
0.035 \times 0.87 & 0.078 \times 0.810 .042 \times 0.840 .033 \times 0.83
\end{array}\right]
\end{aligned}
$$

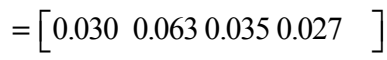

Similarly the weighted composite cloud center-of-gravity vector in the ideal state and the actual weighted composite cloud center-of-gravity vector of the other nine second-level indexes could be obtained as follows [4].

Economic effectiveness cloud center-of-gravity vector is:

$$
\begin{aligned}
& G_{12}^{0}=\left[\begin{array}{ll}
0.009 & 0.016
\end{array}\right] \\
& G_{12}=\left[\begin{array}{ll}
0.007 & 0.013
\end{array}\right]
\end{aligned}
$$


Time effectiveness cloud center-of-gravity vector is:

$G_{13}^{0}=\left[\begin{array}{ll}0.042 & 0.030\end{array}\right]$

$G_{13}=\left[\begin{array}{ll}0.035 & 0.252\end{array}\right]$

Network function cloud center-of-gravity vector is:

$G_{21}^{0}=\left[\begin{array}{lll}0.045 & 0.046 & 0.034\end{array}\right]$

$G_{21}=\left[\begin{array}{lll}0.040 & 0.037 & 0.029\end{array}\right]$

Data center cloud center-of-gravity vector is:

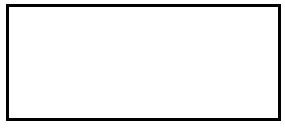

Cloud platform cloud center-of-gravity vector is:

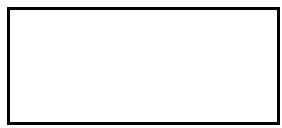

Usable function cloud center-of-gravity vector is:

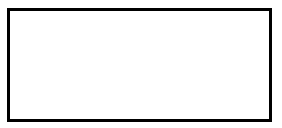

Integrality cloud center-of-gravity vector is:

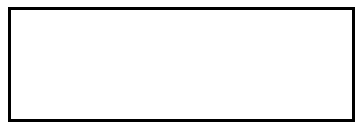
is:

Information relationship cloud center-of-gravity vector

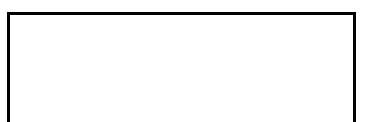

Information usable cloud center-of-gravity vector is:

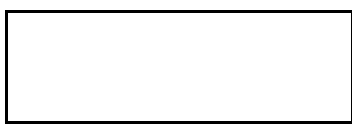

After normalization of $\square$, the data can be got as follows:

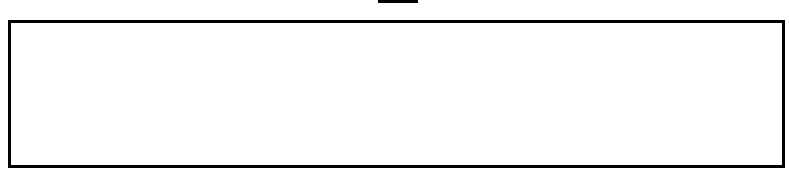

Similarly the other vectors could be normalized as follows:

$$
\begin{array}{ll}
G_{12}=\left[\begin{array}{ll}
-0.18 & -0.18
\end{array}\right] & G_{12}^{\prime}=\left[\begin{array}{lll}
-0.16 & -0.16
\end{array}\right] \\
G_{21}=\left[\begin{array}{lll}
-0.11 & -0.09-0.14
\end{array}\right] & G_{22}^{\prime}=\left[\begin{array}{lll}
-0.11 & -0.13
\end{array}\right] \\
G_{23}=\left[\begin{array}{lll}
-0.16 & -0.16
\end{array}\right] & G_{31}^{\prime}=\left[\begin{array}{lll}
-0.15 & -0.10
\end{array}\right] \\
G_{32}=\left[\begin{array}{lll}
-0.13 & -0.18-0.18
\end{array}\right] & G_{41}=\left[\begin{array}{lll}
-0.15 & -0.17-0.14
\end{array}\right] \\
G_{42} & =\left[\begin{array}{lll}
-0.16 & -0.19 & -0.18
\end{array}\right]
\end{array}
$$

From above, the weighted degree of deviation could be calculated as follows: $\delta_{11}=-0.092, \delta_{12}=-0.005, \delta_{13}=-0.012$

$\delta_{21}=-0.014, \delta_{22}=-0.009, \delta_{23}=-0.012$

$\delta_{31}=-0.009, \delta_{32}=-0.012$

$\delta_{41}=-0.012, \delta_{42}=-0.020$

And the attribution degree of the second-level indexes can be obtained from the formula (8), as follows:

The above results gives an assessed value of the firstlevel indexes product of their weights, as follows:

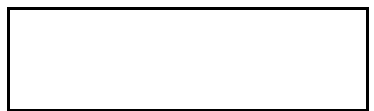

So the total assessment value is $\mathrm{A}=0.962$.

\section{CONCLUSION}

(1) After the assessed value being entered into the cloud generator, two values of the "much better" and "excellent" reviews will be activated, and more inclined to "excellent". Therefore, the efficiency of POL support based on the internet of the things can be identified as "excellent".

(2) The process is feasible and simple, strong operability proved by instance, can integrate the fuzzy ability with randomness to construct the mapping of qualitative and rationality, and offers useful reference for effective evaluation of similar POL support system.

(3) The cloud theory can be used to evaluate most of the system's efficiency problems which are composed of the factors of uncertainty and certainty.

\section{CONFLICT OF INTEREST}

The authors confirm that this article content has no conflict of interest.

\section{ACKNOWLEDGEMENTS}

The work is supported by National Philosophy\& Social Science Funds 12FJS004 and key technology of emergency equipment \& industrialization funds CSTC2012GGSFGC00002.

\section{REFERENCES}

[1] Q. Yong, and Y. Chen, "Lifetime of WSN research based on energy balance", 2012 International Conference on Sensor, Measurement and Intelligent materials, vol. 2, pp. 231-235, 2013. 
[2] L. Liang-cai, F. Lin-jun, and W. Peng, "Method of organization performance evaluation based on cloud theory," Systems Engineering, vol. 28, pp. 99-104, 2010.

[3] L. Liang-cai, and D. Carmicheal. "Evaluation method based on cloud theory and utility theory and its application in owner evaluation," Systems Engineering, vol. 28, pp. 39-45, 2010.
[4] J. Wang, W. Xiao, J.L. Zhang, and B. Deng, "An improved performance assessment method based on cloud model," Fire Power and Command Control, vol. 5, pp. 97-99, 2010.

Received: September 22, 2014

Revised: November 04, 2014

Accepted: November 06, 2014

(C) Yuan et al.; Licensee Bentham Open.

This is an open access article licensed under the terms of the Creative Commons Attribution Non-Commercial License (http://creativecommons.org/licenses/by-nc/3.0/) which permits unrestricted, non-commercial use, distribution and reproduction in any medium, provided the work is properly cited. 